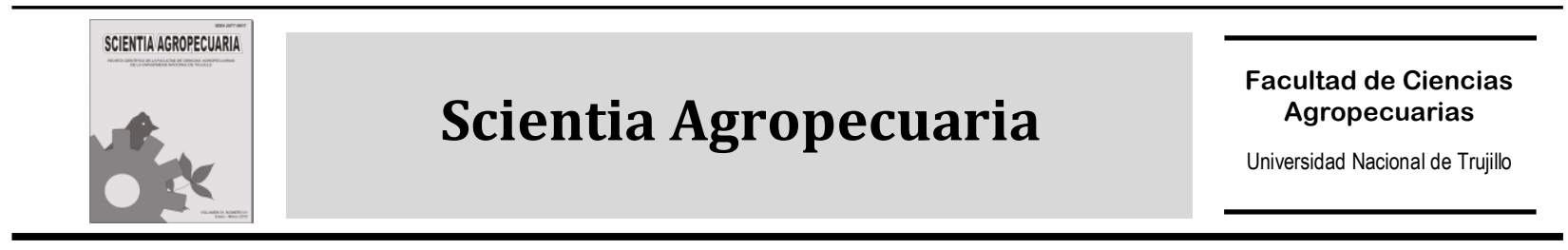

\title{
Efecto de la temperatura y tiempo de transesterificación en el rendimiento y poder calórico de biodiesel a partir de grasa refinada de pollo
}

\section{Effect of transesterification temperature and time on yield and calorific value of biodiesel from refined fat of chicken}

\author{
Hubert Arteaga*, Raúl Siche, Sandra Pagador, Hilda Cáceres \\ Departamento de Ciencias Agroindustriales (Universidad Nacional de Trujillo) Avda. Juan Pablo II s/n Trujillo Perú
}

Recibido 29 enero 2010; aceptado 26 febrero 2010

\begin{abstract}
Resumen
El objetivo principal de esta investigación fue determinar a través de la metodología de superficie de respuesta las condiciones de temperatura y tiempo de transesterificación que permitan obtener el mayor rendimiento y poder calórico en el biodiesel obtenido. Se utilizó el Diseño Compuesto Central Rotacional del tipo $2^{2}+2 * 2$, incluyendo 4 repeticiones en el punto central. La grasa de pollo pasó por procesos de refinación, esterificación y transesterificación a las condiciones de tiempo y temperatura de cada experimento. Las mejores condiciones de temperatura estuvieron entre 50 y $70{ }^{\circ} \mathrm{C}$ y tiempo de transesterificación entre 60 y 120 min, que permitieron obtener rendimientos promedio de $94.5 \%$ y poder calórico en el biodiesel obtenido de $40.2 \mathrm{MJ} / \mathrm{Kg}$. Se concluye que al utilizar la temperatura de $56^{\circ} \mathrm{C}$ y tiempo de 108.4 minutos, se obtiene biodiesel con características exigidas por las normas ASTM D6751 - 07, EN 14214 y NTP 321.003.2005.
\end{abstract}

Palabras clave: Transesterificación, biodiesel, grasa de pollo

\begin{abstract}
The main objective of this research was to determine through the response surface methodology conditions of temperature and time of transesterification which could achieve the higher yield and calorific value in the biodiesel obtained. We used the Central Composite Rotational Design type $2^{2}+2 * 2$, including 4 repetitions at the central point. Chicken fat passed through refining processes, esterification and transesterification weather conditions and temperature of each experiment. The best conditions of temperature were between 50 and $70{ }^{\circ} \mathrm{C}$ and time of transesterification between 60 and $120 \mathrm{~min}$, we have obtained an average yield of $94.5 \%$ and calorific value in the biodiesel obtained from $40.2 \mathrm{MJ} / \mathrm{Kg}$. We conclude that using the temperature of $56^{\circ} \mathrm{C}$ and time of 108.4 minutes, biodiesel is obtained with characteristics required by the ASTM D6751-07, EN 14214 and NTP 321.003.2005 standards.
\end{abstract}

Keywords: transesterification, biodiesel, chicken fat

\section{Introducción}

El biodiesel es un biocombustible hecho de productos naturales renovables como grasas de animales y aceites vegetales. La producción

\footnotetext{
* Autor para correspondencia.

E-mail: hubert_arteaga@yahoo.es (H. Arteaga)
} 
de biodiesel en el Perú está avanzando exitosamente con el desarrollo y prueba de modelos tecnológicos de bajo costo para la producción de biodiesel a pequeña escala a partir de aceites usados y de especies oleaginosas nativas e introducidas (Castro et al., 2007). En Brasil se calcula que se producen pollos de $1.8 \mathrm{~kg}$ en promedio, del cual el $2.4 \%$ es grasa (Intech Engenharia y Medio Ambiente, 2006). Si asumimos que en el Perú se producen pollos con el mismo contenido de grasa, podemos estimar que en la región La Libertad, con una producción de 75.7 millones de aves al año (MINAG, 2006), obtendríamos una cantidad aproximada de 326 mil toneladas de grasa amarilla por año que podrían ser aprovechados para producir biodiesel. Siendo la grasa de pollo un producto de desecho, ésta representa una materia prima potencial para la obtención de biodiesel. En este sentido, este trabajo muestra una opción en la producción de biocombustibles, pero sin utilizar productos alimenticios agrícolas como materia prima, aspecto que está afectando la disponibilidad de recursos alimenticios para la humanidad.

\section{Materiales y métodos}

Fue utilizado grasa de pollo (Gallus gallus) proveniente de Avícola Chimú S.A. Con la finalidad de eliminar impurezas de la grasa de pollo se realizó su refinación (Figura 1).

Se realizó, manualmente, una selección de la grasa a utilizar, separando residuos orgánicos, como: vísceras, tendones, cartílagos, pelos, etc. Para facilitar la extracción de la grasa refinada, la grasa se sometió a una molienda. Luego se calentó la grasa en autoclave a $120^{\circ} \mathrm{C}$ por $60 \mathrm{~min}$. Resultado de este proceso, se obtuvieron dos fases, la grasa derretida en la superficie, y un chicharrón (agua y tejido conjuntivo) en el fondo. Fue extraída la grasa derretida, y luego enfriada. Esto produjo la separación de grasa bruta (flujo de interés), de la gelatina y residuos de agua. La grasa bruta (líquida) fue filtrada a $40^{\circ} \mathrm{C}$ utilizando una tela fina, obteniendo como resultado una grasa líquida refinada. La grasa refinada fue caracterizada en el Laboratorio de Investigación y Química Aplicada de la Universidad Nacional de Ingeniería. Se determinaron parámetros como: índice de acidez y contenidos de ácidos grasos (como el linoleico, oleico, linolénico, entre otros).

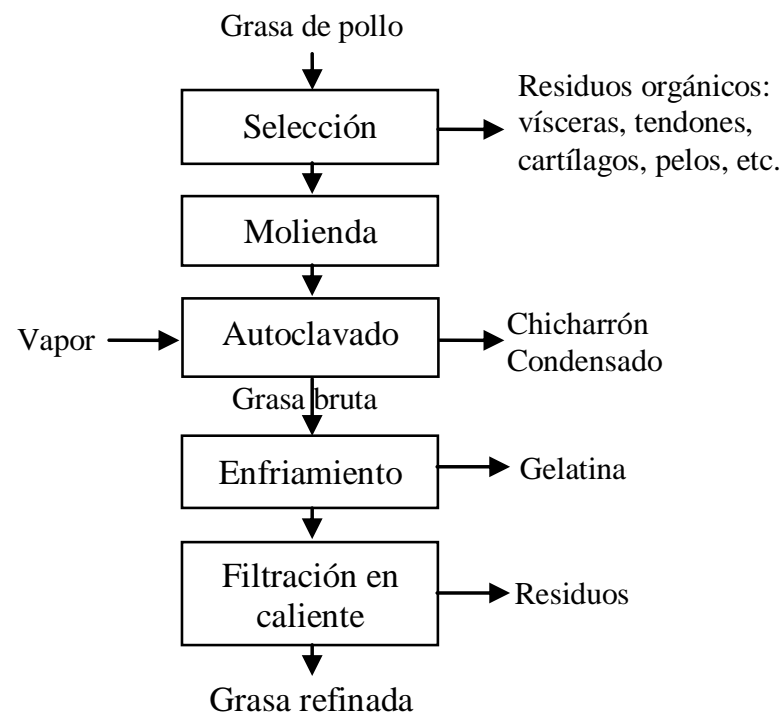

Figura 1. Diagrama de flujo para la obtención de grasa refinada de pollo.

Este proceso de refinamiento asegura que la grasa no tenga sustancias extrañas o sólidas que puedan interferir en el proceso de obtención de biodiesel (Figura 2). La esterificación (Catálisis ácida) de la grasa refinada de pollo se llevó a cabo en un biorreactor con agitación constante a $800 \mathrm{rpm}$, $60^{\circ} \mathrm{C}$ y $150 \mathrm{~min}$. Se utilizó metanol al $20 \% \mathrm{v} / \mathrm{v}$ y ácido sulfúrico como catalizador al $0.21 \%$ v/v (agregado gota a gota). El catalizador $\left(\mathrm{H}_{2} \mathrm{SO}_{4}\right)$ permite que los ácidos grasos libres reaccionen con el metanol formando metilésteres más agua. Este tratamiento se repitió dos veces hasta lograr bajar el índice de acidez a niveles menores que $2 \%$. Finalizada la primera repetición, la mezcla reaccionante se colocó en una pera de decantación durante 8 horas, llevándose a cabo la separación de una fase acuosa (alcohol 
más agua formada durante la reacción) y una fase orgánica (esteres, ácidos grasos aun no esterificados y aceite).

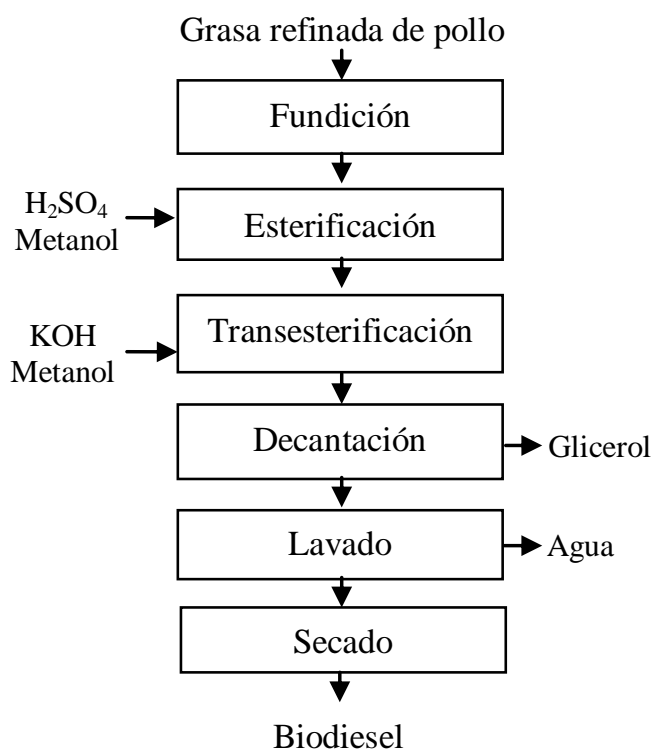

Figura 2. Diagrama de flujo para la obtención de Biodiesel.

En la segunda repetición se tomó la fase orgánica de la primera repetición y se prosiguió como se ha indicado para la primera repetición. En la etapa de transesterificación (Catálisis alcalina) se utilizó el $20 \%$ v/v de metanol respecto a los esteres formados en la catálisis acida y $0.21 \% \mathrm{v} / \mathrm{v}$ de hidróxido de potasio en medias perlas $(90 \% \mathrm{p} / \mathrm{p}$ de pureza) como catalizador, en un biorreactor a $800 \mathrm{rpm}$.

En cada ensayo se utilizó un volumen de 250 $\mathrm{mL}$ de grasa refinada de pollo. El producto obtenido de la transesterificación se dejó decantar durante 24 horas en peras de decantación, tiempo después del cual se procedió a la separación del biodiesel de la glicerina formada. El biodiesel obtenido se lavó cuatro veces, hasta que el agua de lavado se mostró transparente. Por cada litro de biodiesel se usó un litro de agua destilada. Finalmente se realizó un secado en estufa (para eliminar el agua residual) a $105^{\circ} \mathrm{C}$, por aproximadamente $30 \mathrm{~min}$ (hasta que no hubo formación de burbujas de vapor). En la etapa de transesterificación se evaluó el efecto de la temperatura $\left(50\right.$ a $90^{\circ} \mathrm{C}$ ) y el tiempo (40 a 120 min) en el rendimiento y el poder calórico del biodiesel. El biodiesel con mejor rendimiento y poder calórico fue caracterizado en sus principales propiedades físicas e índices característicos de acuerdo con las normas ASTM. Este análisis se realizó por el método de Cromatografía de gases en el Laboratorio de Investigación y Química Aplicada de la Universidad Nacional de Ingeniería.

\section{Análisis estadístico}

Fue utilizado un Diseño Compuesto Central Rotacional (DCCR) de segundo orden con resultados en Superficie de Respuesta. Este diseño, incluye $2^{\mathrm{k}}$ factoriales $(+1,-1), 2 * \mathrm{k}$ puntos axiales $(+1.41,-1.41)$, y cuatro puntos centrales $(0,0)$ para evaluar el error experimental $(\mathrm{k}=2$ variables independientes: temperatura $\mathrm{y}$ tiempo), totalizando 12 tratamientos (Tabla 3). En la tabla 1 se muestran los niveles de las variables independientes ( $x_{1}$ : tiempo; $x_{2}$ : temperatura).

\section{Tabla 1}

Niveles utilizados en el DCCR para las dos variables seleccionadas

\begin{tabular}{cccccc}
\hline Variables & -1.41 & -1 & 0 & 1 & 1.41 \\
\hline$x_{1}$ & 50 & 56 & 70 & 84 & 90 \\
$x_{2}$ & 40 & 51.6 & 80 & 108.4 & 120 \\
\hline
\end{tabular}

Se construyeron modelos del tipo:

$$
Y \approx \beta_{o}+\beta_{1} x_{1}+\beta_{2} x_{2}+\beta_{11} x_{1}^{2}+\beta_{22} x_{2}^{2}+\beta_{12} x_{1} x_{2}
$$

(Donde $\beta_{o}, \beta_{1}, \beta_{2}, \beta_{11}, \beta_{22}, y \beta_{12}$ : Coeficientes de regresión; $Y$ : Respuesta), en función de los coeficientes significativos para cada respuesta (rendimiento y poder calórico). Luego, se realizó un ANVA para los modelos y el cálculo de los coeficientes de determinación $\left(R^{2}\right)$, pruebas que permiten validar estadísticamente los modelos. Finalmente, se generaron superficies de respuesta, en donde se buscaron regiones de interés. 


\section{Resultados y discusión}

\subsection{Caracterización de la grasa de pollo}

En la tabla 2 se muestra la composición química de la grasa de pollo utilizada.

\section{Tabla 2}

Composición química de la grasa refinada de pollo

\begin{tabular}{lcc}
\hline Parámetro & Cantidad & Unidades \\
\hline Linoleico & 20.6 & $\%$ \\
Oleico & 42.5 & $\%$ \\
Palmítico & 21.5 & $\%$ \\
Esteárico & 7.6 & $\%$ \\
Linolénico & 1.8 & $\%$ \\
Otros & 6.1 & $*$ n.d. \\
\hline
\end{tabular}

*n.d.= no determinado

Fuente: Laboratorio de Investigación y Química Aplicada (Universidad Nacional de Ingeniería - Perú)

El índice de acidez de la grasa refinada de pollo fue de $63 \%$. Arango (2002) argumenta que un valor elevado del índice de acidez indica que la grasa contiene una alta cantidad de ácidos grasos libres, ya que ha sufrido un alto grado de hidrólisis. Zhang et al. (2003) argumentan que la determinación del índice de acidez es importante para el proceso de producción de biodiesel (transesterificación), ya que el contenido elevado de ácidos grasos libres lleva a un menor rendimiento en la producción de biodiesel, por la reacción de estos con el catalizador de la transesterificación formándose jabones (saponificación). Esta grasa, por su elevada acidez (mayor a 11\%) no es recomendable en piensos para ganado vacuno, aves o animales jóvenes, por su influencia negativa sobre el consumo y la productividad (Blas et al., 2003). Los ácidos grasos Oleico (42.5\%), Palmítico (21.5\%) y Linoleico $(20.6 \%)$ son los que se encuentran en mayor proporción en esta grasa.

\section{Transesterificación de la grasa de pollo}

La tabla 3 muestra el efecto de la temperatura y tiempo del proceso de transesterificación en el rendimiento y poder calórico del biodiesel. Observando los puntos centrales (tratamientos del 9 al 12), ambas respuestas presentan una pequeña variación, indicando una buena repetibilidad del proceso. Se alcanza un rendimiento mínimo $(73.3 \%)$ en el tratamiento $6\left(90^{\circ} \mathrm{C}\right.$ por 80 minutos) y máximo $(94.5 \%)$ en el tratamiento $3\left(56^{\circ} \mathrm{C}\right.$ por $\left.108.4 \mathrm{~min}\right)$. En el caso del poder calórico, se alcanza un mínimo $(37.5 \mathrm{MJ} / \mathrm{kg})$ en el tratamiento $4\left(84^{\circ} \mathrm{C}\right.$ por $108.4 \mathrm{~min})$ y máximo $(40.2 \mathrm{MJ} / \mathrm{kg})$ en el tratamiento $3\left(56^{\circ} \mathrm{C}\right.$ por $\left.108.4 \mathrm{~min}\right)$.

\section{Tabla 3}

Rendimiento y poder calórico del biodiesel

\begin{tabular}{|c|c|c|c|c|c|c|}
\hline \multirow{2}{*}{$\mathrm{N}$} & \multicolumn{2}{|c|}{$\begin{array}{c}\text { Temperatura } \\
\left({ }^{\circ} \mathrm{C}\right)\end{array}$} & \multicolumn{2}{|c|}{$\begin{array}{c}\text { Tiempo } \\
\text { (min) }\end{array}$} & \multirow{2}{*}{$\begin{array}{c}\mathrm{R} \\
(\%)\end{array}$} & \multirow{2}{*}{$\begin{array}{c}\mathrm{PC} \\
(\mathrm{MJ} / \mathrm{kg})\end{array}$} \\
\hline & $\begin{array}{l}\text { Códi- } \\
\text { go }\end{array}$ & Real & $\begin{array}{l}\text { Códi- } \\
\text { go }\end{array}$ & Real & & \\
\hline 1 & -1 & 56 & -1 & 51.6 & 80.1 & 38.4 \\
\hline 2 & 1 & 84 & -1 & 51.6 & 77.5 & 37.6 \\
\hline 3 & -1 & 56 & 1 & 108.4 & 94.5 & 40.2 \\
\hline 4 & 1 & 84 & 1 & 108.4 & 79.7 & 37.5 \\
\hline 5 & -1.41 & 50 & 0 & 80 & 82.0 & 39.7 \\
\hline 6 & 1.41 & 90 & 0 & 80 & 73.3 & 38.7 \\
\hline 7 & 0 & 70 & -1.41 & 40 & 84.8 & 38.5 \\
\hline 8 & 0 & 70 & 1.41 & 120 & 91.4 & 39.2 \\
\hline 9 & 0 & 70 & 0 & 80 & 88.6 & 39.5 \\
\hline 10 & 0 & 70 & 0 & 80 & 88.5 & 39.4 \\
\hline 11 & 0 & 70 & 0 & 80 & 88.2 & 39.4 \\
\hline 12 & 0 & 70 & 0 & 80 & 88.3 & 39.3 \\
\hline
\end{tabular}

N: Número de tratamiento; R: Rendimiento; PC: Poder calórico

En los tratamientos 1 y 2 , el tiempo permanece fijo en $51.6 \mathrm{~min}$ mientras que la temperatura aumenta de 56 a $84^{\circ} \mathrm{C}$. Bajo estas condiciones, ocurre una disminución del rendimiento desde $80.1 \%$ a $77.5 \%$ y el poder calórico desde $38.4 \mathrm{MJ} / \mathrm{kg}$ a $37.6 \mathrm{MJ} / \mathrm{kg}$. En los tratamientos 3 y 4 , la temperatura se aumenta de 56 a $84^{\circ} \mathrm{C}$ y el tiempo permanece fijo (108.4 min). Bajo estas condiciones también se nota una disminución del rendimiento (de $94.5 \%$ a $79.7 \%$ ) y el poder calórico (de $40.2 \mathrm{MJ} / \mathrm{kg}$ a $37.5 \mathrm{MJ} / \mathrm{kg}$ ). Considerando los tratamientos 1 y 3 (a $56^{\circ} \mathrm{C}$ ), se observa que a medida que el tiempo aumenta (de 51.6 a $108.4 \mathrm{~min}$ ), el rendimiento y el poder calórico también aumentan (de $80.1 \%$ a $94.5 \%$; y de $38.4 \mathrm{MJ} / \mathrm{kg}$ a 40.2 
$\mathrm{MJ} / \mathrm{kg}$, respectivamente). Sin embargo para los tratamientos 5 y 9 , donde la temperatura va de 50 a $70^{\circ} \mathrm{C}$ (ambos tratamientos evaluados a $80 \mathrm{~min})$, el rendimiento aumenta $(82 \%$ a $88.6 \%)$ y el poder calórico disminuye ligeramente $(39.7 \mathrm{MJ} / \mathrm{kg}$ a $39.5 \mathrm{MJ} / \mathrm{kg})$. Estos resultados indican que la temperatura tiene un efecto positivo en el rendimiento hasta temperaturas cercanas de $70^{\circ} \mathrm{C}$, pero al superar esta temperatura el rendimiento decae. Torossi (2006) precisa que la reacción de transesterificación puede ocurrir a $25^{\circ} \mathrm{C}$, y su incremento es directamente proporcional a la velocidad de reacción, sin embargo posee un intervalo limitado por el punto de ebullición del metanol $\left(65^{\circ} \mathrm{C}\right)$ y por las reacciones secundarias de formación de jabones debiéndose mantenerse entre 25 y $65^{\circ} \mathrm{C}$. Rojas et al. (2009) también indican que la transesterificación depende del aceite $\mathrm{y}$ alcohol empleado y que al aumentar la temperatura, aumenta el rendimiento y el tiempo de reacción se reduce. De esta forma se recomienda que la temperatura no exceda el punto de ebullición del alcohol, porque se vaporiza y forma burbujas que limitan la reacción en la interfase alcohol/aceite/ biodiesel, y que el rendimiento aumenta con el tiempo de reacción.

El poder calórico tiene un comportamiento similar al rendimiento en función de la temperatura y tiempo de transesterificación, y presenta una variación no tan marcada entre cada tratamiento oscilando entre $37.5 \mathrm{MJ} / \mathrm{kg}$ y $40.2 \mathrm{MJ} / \mathrm{kg}$ (Figura 3), por lo que el efecto de la temperatura y tiempo está supeditado al tipo de grasa a utilizar. Mittelbach (1996) indica que el poder calórico depende del aceite utilizado más no del proceso de producción, reporta que la grasa refinada de pollo tiene un poder calorífico de $39.8 \mathrm{MJ} / \mathrm{kg}$. Para el Rendimiento, todos los coeficientes de regresión resultan ser significativos $(p<0.05)$ para el modelo, a excepción del tiempo $(\mathrm{Q})$ (Tabla 4); mientras que para el poder calórico, todos los coeficientes son significativos.

\section{Tabla 4}

Coeficientes de Regresión para el Rendimiento y Poder Calórico

\begin{tabular}{lrrrrr}
\hline \multirow{2}{*}{ Ítem } & \multicolumn{2}{c}{ Rendimiento } & & \multicolumn{2}{c}{ Poder calórico } \\
\cline { 2 - 3 } \cline { 5 - 6 } & Coeficiente & $p$ & & Coeficiente & $p$ \\
\hline Intercepto & -78.35 & $5.9 \mathrm{E}-05$ & & 25.79 & $4.0 \mathrm{E}-04$ \\
$x_{1}(\mathrm{~L})$ & 4.12 & $5.1 \mathrm{E}-06$ & & 0.21 & $7.7 \mathrm{E}-03$ \\
$x_{I}(\mathrm{Q})$ & -0.027 & $5.5 \mathrm{E}-06$ & & -0.0011 & $1.5 \mathrm{E}-02$ \\
$x_{2}(\mathrm{~L})$ & 0.68 & $7.2 \mathrm{E}-05$ & & 0.18 & $9.0 \mathrm{E}-04$ \\
$x_{2}(\mathrm{Q})$ & -0.0002 & $1.4 \mathrm{E}-01$ & & -0.0005 & $2.6 \mathrm{E}-03$ \\
$x_{1}(\mathrm{~L}) * x_{2}(\mathrm{~L})$ & -0.008 & $5.9 \mathrm{E}-05$ & & -0.0012 & $3.1 \mathrm{E}-03$ \\
\hline
\end{tabular}

$x_{1}$ : temperatura; $x_{2}$ : tiempo; $(\mathrm{L})=$ lineal; $(\mathrm{Q})=$ cuadrática

Los modelos matemáticos para las respuestas $y_{1}$ (rendimiento) y $y_{2}$ (poder calórico) quedaron como sigue:

$$
\begin{gathered}
y_{1}=-78.35+4.12 x_{1}-0.027 x_{1}^{2}+0.68 x_{2}-0.008 x_{1} x_{2} \\
y_{2}=25.79+0.21 x_{1}-0.001 x_{1}^{2}+0.18 x_{2}-0.0005 x_{2}^{2}- \\
0.0012 x_{1} x_{2}
\end{gathered}
$$

Note, que para la respuesta $y_{1}$ (Rendimiento) no se ha considerado el coeficiente del tiempo (Q), ya que no resulta ser significativo $(\mathrm{p}=0.14>0.05)$ para el modelo.

\section{Análisis de varianza}

La tabla 5 muestra el análisis de varianza para los modelos de regresión, tanto para el rendimiento como para el poder calórico.

\begin{tabular}{|c|c|c|c|c|c|c|c|c|c|}
\hline $\begin{array}{l}\text { Variable } \\
\text { respuesta }\end{array}$ & $\begin{array}{l}\text { Fuente de } \\
\text { variación }\end{array}$ & $\begin{array}{l}\text { Grados } \\
\text { Libertad }\end{array}$ & $\begin{array}{l}\text { Suma de } \\
\text { cuadrados }\end{array}$ & $\begin{array}{c}\text { Cuadrados } \\
\text { medios }\end{array}$ & $\mathrm{F}_{\text {calculado }}$ & $\begin{array}{c}\mathrm{p}- \\
\text { valor }\end{array}$ & $F_{\text {tabla }}$ & $\mathrm{R}^{2}$ & $\begin{array}{c}\mathrm{R}^{2} \\
\text { ajustado }\end{array}$ \\
\hline \multirow{3}{*}{ Rendimiento } & Regresión & 4 & 431.28 & 107.81 & 72.81 & $<0.05$ & 4.20 & $97.6 \%$ & $95.7 \%$ \\
\hline & Residuos & 7 & 10.36 & 1.48 & & & & & \\
\hline & Total & 11 & 441.59 & 40.14 & & & & & \\
\hline \multirow{3}{*}{$\begin{array}{l}\text { Poder } \\
\text { calórico }\end{array}$} & Regresión & 5 & 6.31 & 1.26 & 5.44 & $<0.05$ & 4.39 & $81.9 \%$ & $66.8 \%$ \\
\hline & Residuos & 6 & 1.39 & 0.23 & & & & & \\
\hline & Total & 11 & 7.70 & 0.70 & & & & & \\
\hline
\end{tabular}

Tabla 5

ANVA para el Rendimiento y poder calórico 
Se puede observar que ambos modelos resultan ser significativos $\left(F_{\text {tabla }}<F_{\text {calculado }}\right)$, aunque el modelo para el rendimiento resulta ser altamente significativo (4.20 $<71.11)$, mientras que el modelo para el poder calórico es levemente significativo $(4.39<5.44)$. Así, para determinar si ambos modelos son adecuados, se tuvo que analizar, además de la significancia, el coeficiente de determinación $\left(\mathrm{R}^{2}\right)$. Para el rendimiento, se alcanza un $\mathrm{R}^{2}$ de 97.6\% ( $\mathrm{R}^{2}$ ajustado: 95.7\%), indicando un buen ajuste del modelo, es decir, casi el $98 \%$ de la variabilidad del rendimiento es explicado por el modelo (con las variables estudiadas y en los niveles establecidos). Sin embargo, para el poder calórico, se obtuvo un $\mathrm{R}^{2}=81.9 \%$ ( $\mathrm{R}^{2}$ ajustado: $66.8 \%$ ), indicando un modelo con un bajo nivel de explicación. De lo anterior se concluye, que es recomendable analizar el rendimiento vía su superficie de respuesta, mas no sería adecuado hacer lo mismo para el poder calórico.

\section{Análisis por superficie respuesta}

Observando la figura 3, las condiciones que permiten maximizar el rendimiento $(94.5 \%)$ son: temperatura desde $55^{\circ} \mathrm{C}$ a $70^{\circ} \mathrm{C}$ y tiempos desde 90 a 120 min. Para validar las condiciones óptimas para el rendimiento, se realizaron dos corridas experimentales (Tabla 6). Se puede observar que los desvíos relativos, de la comparación entre los valores experimentales y previstos por el modelo son muy pequeños (menor a $2 \%$ ), por lo que el modelo resulta muy adecuado para predecir las condiciones óptimas de rendimiento de biodiesel.

\section{Tabla 6}

Valores de rendimiento experimental y previsto por el modelo

\begin{tabular}{cccccc}
\hline $\begin{array}{c}\text { Trata- } \\
\text { miento }\end{array}$ & $\begin{array}{c}\text { Tempera- } \\
\text { tura }\end{array}$ & Tiempo & $\begin{array}{c}y_{1} \\
\text { experimental }\end{array}$ & $\begin{array}{c}y_{l} \\
\text { previsto }\end{array}$ & $\begin{array}{c}\text { Desvío } \\
(\%)\end{array}$ \\
\hline 1 & 56 & 108.4 & 94.5 & 92.8 & 1.8 \\
2 & 70 & 120 & 91.4 & 92.2 & 0.8 \\
\hline
\end{tabular}

Por otro lado, como el modelo para el poder calórico no fue adecuado estadísticamente, pero tomando como referencia las condiciones que maximizan el rendimiento, podemos asegurar que bajo condiciones de $56^{\circ} \mathrm{C}$ y 108.4 min se obtiene, coincidentemente, un máximo poder calórico de $40.2 \mathrm{MJ} / \mathrm{kg}$ (Tabla $3)$.

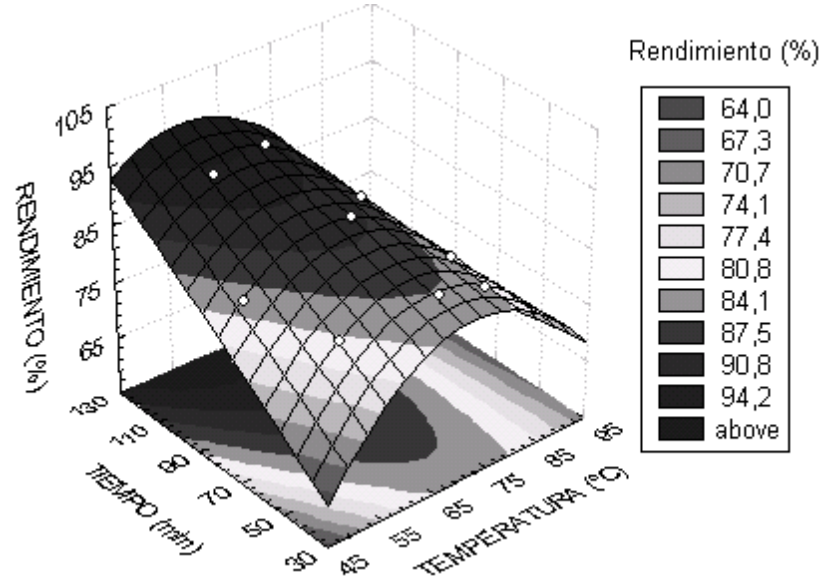

(a)

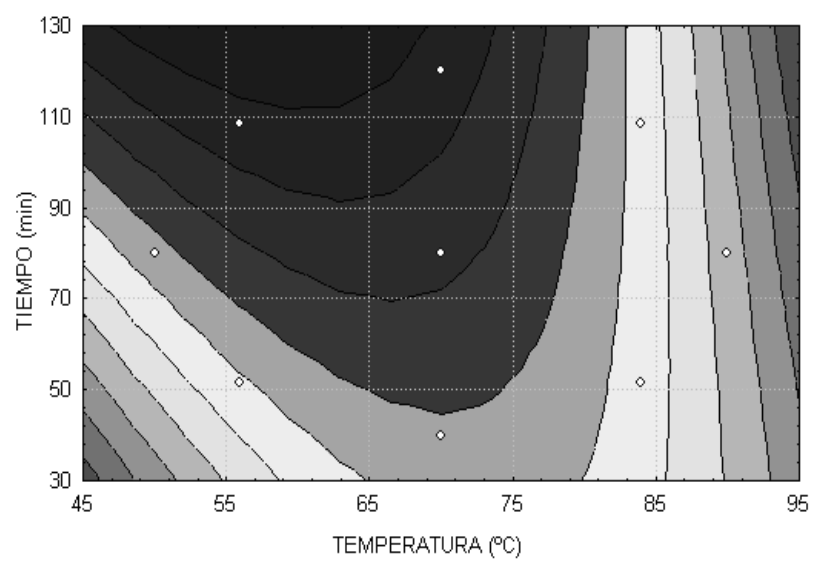

(b)

Figura 3. (a) Superficie de Respuesta; (b) curvas de contorno para el Rendimiento en función de la temperatura y tiempo. 


\section{Caracterización del biodiesel con mayor rendimiento y poder calórico}

La calidad del biodiesel es generalmente controlada en base a ciertos parámetros físicos y químicos establecidos por normas técnicas aprobadas en cada país. Según Acosta (2006), las principales normas técnicas para el biodiesel son la ASTM D6751 - 07, EN 14214 y NTP 321.003 2005. La tabla 7, muestra las características del biodiesel con mayor rendimiento y poder calórico.

\section{Tabla 7}

Características del biodiesel con mayor rendimiento y poder calórico

\begin{tabular}{lr}
\hline Características & Cantidad \\
\hline Punto de inflamación, ${ }^{\circ} \mathrm{C}$ & 176.5 \\
Punto inicial de ebullición, ${ }^{\circ} \mathrm{C}$ & 190 \\
$90 \%$ Recuperación, ${ }^{\circ} \mathrm{C}$ & 287 \\
$95 \%$ Recuperación, ${ }^{\circ} \mathrm{C}$ & 255 \\
Punto final de ebullición, ${ }^{\circ} \mathrm{C}$ & 312 \\
Densidad $\left(15^{\circ} \mathrm{C}\right), \mathrm{g} / \mathrm{mL}$ & 0.8643 \\
Viscosidad Cinemática $\left(40^{\circ} \mathrm{C}\right), \mathrm{mm}^{2} / \mathrm{s}$ & 4.53 \\
Agua y Sedimentos, $\% ~ V o l$. & $<0.01$ \\
Índice de Cetano & 60.08 \\
Índice de Acidez $\mathrm{mg} \mathrm{KOH} / \mathrm{g}$ & 0.32 \\
Cenizas sulfatadas $\%(\mathrm{~m} / \mathrm{m})$ & 0.01 \\
Glicerina Libre $\%(\mathrm{~m} / \mathrm{m})$ & 0.01 \\
Glicerina Total $\%(\mathrm{~m} / \mathrm{m})$ & 0.15 \\
Residuo carbonoso $\%(\mathrm{~m} / \mathrm{m})$ & $<0.05$ \\
\hline
\end{tabular}

Fuente: Laboratorio de Investigación y Química Aplicada (UNI)

El punto de inflamación obtenido en el biodiesel es de $176.5^{\circ} \mathrm{C}$, valor superior al estándar permitido por la Norma ASTM (130 ${ }^{\circ} \mathrm{C}$ ), lo que permite, según Castro et al. (2007), garantizar que se haya removido todo el metanol que afecta a las bombas de combustible, sellos y empaquetaduras, y provocar una mala combustión.

La destilación al $90 \%$ y $95 \%$ de recuperación fue de $287^{\circ} \mathrm{C}$ y $255^{\circ} \mathrm{C}$, valores se encuentran en el rango establecido por la Norma ASTM y la Norma Europea que indican un máximo de $360^{\circ} \mathrm{C}$; así, este biodiesel, tiene un punto de destilación que se ubica en el rango más alto de la curva del diesel, parámetro que se incorpora para controlar que el combustible no haya sido contaminado con materiales de mayor punto de evaporación (Castro et al., 2007).

La densidad a $15^{\circ} \mathrm{C}$ fue de $0.8643 \mathrm{~g} / \mathrm{mL}$, valor dentro del rango establecido por la Norma Europea $(0.86-0.90 \mathrm{~g} / \mathrm{mL})$. No existe en la Norma ASTM regulación de la densidad para el biodiesel.

La viscosidad cinemática $\left(\mathrm{a} 40^{\circ} \mathrm{C}\right.$ ) fue de 4.53 $\mathrm{mm}^{2} / \mathrm{s}$, valor dentro de los rangos establecidos por las Normas ASTM $\left(1.9-6.0 \mathrm{~mm}^{2} / \mathrm{s}\right)$ y Europea $\left(3.5-5.0 \mathrm{~mm}^{2} / \mathrm{s}\right)$. Castro et al. (2007) indican que la viscosidad del biodiesel viene determinada por el aceite de origen, y por su contenido en mono, di y triglicéridos; una transesterificación completa es necesaria para asegurar el cumplimiento de este parámetro; un combustible muy viscoso puede causar una mala atomización, que lleva a mala combustión y formación de depósitos, la alta viscosidad también puede facilitar la contaminación del combustible con el aceite lubricante.

El porcentaje de agua y sedimentos es menor a $0.01 \% \mathrm{v} / \mathrm{v}$, valor muy por debajo del estándar establecido por las Normas (máximo $0.05 \%$ $\mathrm{v} / \mathrm{v})$. Esta característica, permite obtener un mayor rendimiento y mejor calidad de biodiesel. Según Romano (1982), la presencia de agua $(0.1 \% \mathrm{v} / \mathrm{v})$ es un indicador suficiente para reducir la producción de esteres y de glicerina. Por otro lado, Castro et al. (2007) indican que técnicas inadecuadas de secado del biodiesel o contacto con agua durante el transporte pueden afectar su calidad; el biodiesel es muy higroscópico y puede absorber agua si es almacenado en contacto con el aire húmedo, o durante las operaciones de carga y descarga, por lo que es recomendable almacenarlo bajo una atmósfera inerte (nitrógeno) o tener tanques con techo flotante. Los sedimentos pueden aparecer debido a la oxidación del combustible, así que este análisis, junto con los de acidez y viscosidad ayudan a establecer si el combustible se ha oxidado durante su 
almacenamiento. El agua también puede generar corrosión y promueve el desarrollo de microorganismos, y los sedimentos pueden causar problemas de taponamiento de filtros e inyectores.

El índice de cetano fue de 60.08 , valor por encima del mínimo establecido por la norma ASTM (47 mínimo) y Europea (51 mínimo). Blanco (2000) precisa que se requiere de un índice de cetano adecuado para un buen desempeño del motor. Valores altos del índice de cetano mejoran el arranque en frío y minimizan la formación de humo blanco. También indica que este valor depende del tipo de materia prima (distribución de ácidos grasos) y nivel de oxidación del biodiesel.

El índice de acidez fue de $0.32 \mathrm{mg} \mathrm{KOH} / \mathrm{g}$. La norma ASTM y europea reportan para este parámetro un valor de $0.50 \mathrm{mg} \mathrm{KOH} / \mathrm{g}$ como máximo. Castro et al. (2007) indican que valores altos de índice de acidez se deben a la presencia de ácidos grasos libres en el biodiesel, debido a una producción inadecuada y al combustible degradado durante su almacenamiento.

El porcentaje de cenizas sulfatadas fue de $0.01 \% \mathrm{~m} / \mathrm{m}$. Según la norma ASTM y europea, el porcentaje de cenizas debe ser como máximo $0.02 \% \mathrm{~m} / \mathrm{m}$. Estas dependen principalmente de la cantidad de catalizador residual presente en el biodiesel, o de cualquier otro compuesto que produzca cenizas (jabones y sólidos abrasivos). El lavado del biodiesel asegura el cumplimiento de este parámetro (Castro et al., 2007).

La glicerina libre obtenida fue de $0.01 \% \mathrm{~m} / \mathrm{m}$ y según las normas debe tener un máximo de $0.02 \% \mathrm{~m} / \mathrm{m}$. La glicerina libre indica presencia de glicerol residual en el biodiesel, debido a deficiencia en su lavado o purificación (Castro et al., 2007). La glicerina total fue de $0.15 \% \mathrm{~m} / \mathrm{m}$ y según la norma europea debe ser máximo $0.25 \% \mathrm{~m} / \mathrm{m}$. La glicerina total indica presencia de glicerol residual y de mono, di y triglicéridos, debido a una transesterificación incompleta (Castro et al., 2007).

El residuo carbonoso reporto valores menores de $0.05 \% \mathrm{~m} / \mathrm{m}$. La norma ASTM indica un valor máximo de $0.2 \% \mathrm{~m} / \mathrm{m}$ para esta característica. García y García (2006) mencionan que el residuo carbonoso es una medida de la tendencia del combustible a formar depósitos carbonosos en el motor a partir de algunas impurezas como glicéridos, ácidos grasos, jabones y restos de catalizador.

\section{Conclusiones}

La temperatura en los niveles estudiados tiene un efecto significativo en el rendimiento $\mathrm{y}$ poder calórico. Este efecto es positivo hasta temperaturas cercanas al punto de ebullición del metanol. Superado esta temperatura el efecto es negativo. El tiempo en los niveles estudiados tiene un efecto significativo positivo tanto en el rendimiento como en el poder calórico. A través de la metodología de superficie de respuesta se maximizó el rendimiento $(94.5 \%)$ y el poder calórico (40.2 $\mathrm{MJ} / \mathrm{Kg}$ ) en el biodiesel obtenido a partir de la grasa refinada de pollo (Gallus gallus), a condiciones de temperatura entre $50 \mathrm{y} 70^{\circ} \mathrm{C}$, y tiempo de transesterificación entre 60 y 120 minutos. Al utilizar una combinación de $56^{\circ} \mathrm{C}$ de temperatura y un tiempo 108.4 minutos, en la etapa de transesterificación, se obtiene un biocombustible, a partir de la grasa de pollo, de excelente calidad ya que cumple con todas las normas establecidas por las normas ASTM y Europeas.

\section{Referencias}

Acosta, L. 2006. Proyecto Biodiesel UNALM / ITDG. I Congreso sobre Biocombustibles y Energías Renovables. Experiencias en el Laboratorio de Energías Renovables: 11- 14

Blanco, M. 2000. Química Biológica. Sexta edición Disponible en: http://www.taringa.net/posts/info/ 1001745/Biodiésel.html

Blas, C.; Mateos, G.G.; Rebollar, P.G. (Eds.). 2003. Tablas FEDNA de composición y valor nutritivo de alimentos para la formulación de piensos compuestos. $2^{\mathrm{a}}$ ed. Fundación Española para el Desarrollo de la Nutrición Animal. Madrid, España. 423 pp. 
Castro, P.; Coello, J.; Castillo, L. 2007. Opciones para la producción y uso del biodiesel en el Perú- Lima: Soluciones Prácticas-ITDG. Primera edición: 10-30.

García, J.; García, J. 2006. Informe de vigilancia tecnológica, Biocarburantes líquidos: biodiesel y bioetanol. CEIM. Disponible en: http://www.madrimasd.org/ informacionIDI/biblioteca/Publicacion/doc/VT/vt4_Bioca rburantes_liquidos_biodiesel_y_bioetanol.pdf

Intech Engenharia y Medio Ambiente, 2006. Biodiesel con grasa de pollo. Disponible en: http://www.engormix.com/ s_news9464.htm

MINAG, 2006. Producción pecuaria. Disponible en: http://www.minag.gob.pe/pecuaria/situacion-de-lasactividades-de-crianza-y-produccion-4.html

Mittelbach, M. 1996. Diesel fuel derived from vegetable oils, VI: Specifications and quality control of biodiesel. Bioresource Technology 56: 7-11.

Romano, 1982. Vegetables oils: a new alternative. Proc.Int.Conf.on Plant and Vegetables oils as Fuels. 10116. Fargo, 2-4 Agust. St. Joseph, Mich.: ASAE.
Rojas, A.; Girón, E.; Torres, H. 2009. Variables de operación en el proceso de transesterificación de aceites vegetales: una revisión - catálisis química. Revista Ingeniería e Investigación, 29(3): 17-22. Disponible en: http://www.revistaingenieria.unal.edu.co/Resumenes/29_3 13_869.pdf

Torossi, F. 2006. Reacciones en contexto: la transesterificación en la producción de biodiesel a partir de aceite de fritura usado. Revista ANALES de la Real Sociedad Española de Química, 102 (3): 43-49. Disponible en: http://www.dialnet.unirioja.es/servlet/ fichero_articulo?codigo $=2082917 \ldots 0$

Zhang, Y.; Dubee, M.; Mclean, D.; Kates, M. 2003. Biodiesel production from waste cooking oil: 1 . Process design and technological assessment. Bioresource Technology 1-16. 